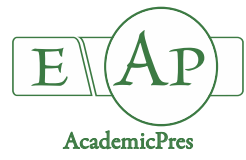

\title{
Induction of Lateral Branching of Sweet Cherry and Plum in Fruit Nursery
}

\author{
Jan WOLF*, Tomáš KISS, Ivo ONDRÁŠEK, Tomáš NEČAS \\ Mendel University in Brno, Faculty of Horticulture in Lednice, Department of Fruit Growing, Valtická 337, Lednice, 69144, Czech Republic; \\ jan.wolf@mendelu.cz(*correspondingauthor); tomas.kiss@mendelu.cz;ivo.ondrasek@mendelu.cz; tomas.necas@mendelu.cz
}

\begin{abstract}
Modern high quality nursery trees pruning as knipboom grow and crop significantly better during the first years after planting in the orchard. High quality nursery trees perform better in the first years after plantation in the orchard than average or low quality nursery trees. Nurserymen are focused on production of such trees in the shortest time (one year). However, fruit tree species and varieties differ in their abilities to produce these trees. The goal of this work was to increase the quality and branching of one-year-old nursery trees at two plum and two sweet cherry varieties by the treatments of 12 different preparations of plant growth regulators (PGRs). Before lifting, branching and trunk parameters were recorded. Data showed that Ethephone+Putrescine+ $+\mathrm{GA}_{4+7}$ and Ethephone+Putrescine+6-BAP significantly increased almost all parameters at both plum varieties. Both sweet cherry varieties were sufficiently branching with Globaryl+epibrassinolide treatment, however the lateral shoots of 'Celeste' sweet cherry variety were of low quality. The same was observed after treatment with Progerbalin+4CPPU+Putrescine. Sweet cherry 'Kordia' branched very well after Globaryl and TIBA+PBZ treatments too, however, the lateral shoots after treatment with the latter preparation were of low quality. 'Kordia' trees after Globaryl treatment were branched in $94 \%$ of cases with more than four lateral shoots.
\end{abstract}

Keywords: apical dominance; feathering; PGRs; Prunus avium; Prunus domestica

\section{Introduction}

The quality of planting material has great effect on an early fruit onset and profitability of high density planting systems. A good root system, strong stem, and well developed crown consisting plentiful lateral shoots with wide crotch angles distributed along the main shoot are characteristic for high quality young trees. Such trees are required by growers for new plantations as they grow better (Preston, 1968) and crop more heavily during first years in the orchard (Zahn, 1996). Nurseries are, thus, improving their cultivation technologies to meet the requested quality of young trees.

Sweet cherries are well known for heterogeneous formation of lateral shoots in nurseries. The capability of young sweet cherry trees to produce lateral shoots depends on the variety. Varieties with weaker apical dominance have better branching characteristics than varieties with strong apical dominance, which form only low amount of lateral shoots (Hrotkó et al., 1999). Compared to sweet cherries, young plum trees branch relatively well in nurseries, however, the heterogeneous formation of lateral shoots between varieties could be also observed. In recent years, nurseries, especially in case of sweet cherries, are focused on improvement of the management to efficiently induce lateral branching (Sazo and Robinson, 2011).

Apart from a knipboom system, which adds higher cost due to an extra year of cultivation in the nursery, one-yearold nursery tree production system could be used to produce well branched trees as well. Here, pinching and chemical agents are used to induce lateral branching (Looney and Jackson, 2011). To lower the price of the nursery tree, nurserymen prefer to use chemical agents (Looney and Jackson, 2011). From these, the best results have been obtained from application of cytokinins and gibberelins.

Studies proved positive effect of 6-benzylaminopurine (6-BAP, cytokinin) and gibberellic acids ${ }_{4+7}\left(\mathrm{GA}_{4+7}\right.$, gibberellins) in different concentrations and doses on branching of sweet cherry (Hrotkó et al., 1999; Elfving and Visser, 2006; Koyuncu and Yidirim, 2008) and plum trees (Magyar and Hrotkó, 2002). GA $4+7$ and 6-BAP have been commercially available in combination as Perlan, Promalin or Progerbalin LG and also separately as Paturyl (6-BAP), Globaryll (6-BAP) or Gibb plus $\left(\mathrm{GA}_{4+7}\right)$. Besides nursery production, 6-BAP is used as fruit thinning agent and $\mathrm{GA}_{4+7}$ to reduce the russet development on apples, while their combination is used to increase the size of apple or pear fruits (Looney and Jackson, 2011). 
In recent decades cyclanilide, which acts as an auxin transport inhibitor (Pedersen et al., 2006), was tested as a promising branching agent of sweet cherry and apple trees (Elfving and Visser, 2006; Sazo and Robinson, 2011). Among plant growth regulators (PGR's) with inhibition effect, paclobutrazol (PBZ, anti-giberrelin) was used together with Promalin to produce compact and well branched sweet cherry trees (Jacyna et al., 1989).

Therefore, PGR's with different impact on growth could be used for production of well branched nursery trees. Curently, a numerous PGR's with these and other effects have been used or tested in fruit production and could be beneficial in nursery production too. In this study, well known branching agents and up-to-date not tested PGR's are tested in their branching abilities with aim to produce well branched high quality one-year-old nursery trees of sweet cherries and plums. Growth inducing PGR's $\mathrm{GA}_{4+7}$, 6-BAP, 24-eppibrassinolide (EBR), 4-CPPU; phytohormone inhibitors 2,3,5-Triiodobenzoic acid (TIBA), naphthylphtalamic acid (NPA), PBZ; stress tolerance promotion agent putrescine $\mathrm{HCl}$ (Put) and ethephon as an ethylene production agent were tested.

\section{Materials and Methods}

\section{Fruit tree nursery description}

The experiment was established in 2017 in a fruit tree nursery (Ökoplant International, Slup) located in the South Moravian Region, Czech Republic. The production area of the nursery is each year located in a new field. Weed within and between the rows was removed and trees were fertilized regularly during the year. If necessary, drum irrigation was used to compensate water deficiency. The average temperature and total rainfall in 2017 were $10.9^{\circ} \mathrm{C} \mathrm{(min.} \mathrm{-}$ $16.6^{\circ} \mathrm{C}$, max. $40^{\circ} \mathrm{C}$ ) and $454.08 \mathrm{~mm}$, respectively.

\section{Plant material}

Two plum and two sweet cherry varieties were selected for the experiment. From plum varieties, the semi-vigorous variety 'Toptaste', a representative of well-branching variety and a vigorous variety 'Lipnická', a representative of poorly branching variety were tested. From the sweet cherries, a relatively well-branching semi-vigorous variety 'Kordia' and a poorly branching and poorly growing variety 'Celeste' were tested. Plums were grafted on St. Julien a rootstock and sweet cherries on Gizela 5 rootstocks.

\section{Preparation and application of $P G R s$}

Three commercially available preparations (GIBB plus $\mathrm{GA}_{4+7} 10 \mathrm{~g}^{-1} \mathrm{l}^{-1}$, L. Gobbi, IT; Globaryl 100 - 6-BAP $100 \mathrm{~g}^{-1} \mathrm{l}^{-1}$, GLOBACHEM, B and Progerbalin LG - GA G $_{4+7} 18.8$ g. $.^{-1}+$ 6-BAP $18.8 \mathrm{gll}^{-1}$, GLOBACHEM B) and 9 other PGRs (EBR, ethephon - Sigma-Aldrich, USA; 4-CPPU, TIBA, NPA, PBZ, GA $4+7$, putrescine, 6-BAP - Duchefa, N) were applied alone or in mixtures in various concentrations (Table 2). Twelve different preparations were prepared for the test (Table 2). In the spring, when the grafted trees reached height of $60 \mathrm{~cm}$, the upper part of the growing terminal of each tree was sprayed with selected preparation. The $60 \mathrm{~cm}$ height was selected according to the practice of the nurseryman, as all side branches up to height of $60 \mathrm{~cm}$ were removed during the vegetation. Plum trees were treated in two terms (22 ${ }^{\text {nd }}$ May and 8th June). The second treatment was applied only on 'Lipnická' variety since the 'Toptaste' variety was sufficiently branched after the first application. Sweet cherry trees were treated three times $\left(8^{\text {th }}\right.$ June, $20^{\text {th }}$ June and 4th July). Each preparation was sprayed with a hand sprayer on a non-windy day and contained 0.5 ml. $\mathrm{l}^{-1}$ of Tween 20 as a surfactant. Each preparation was applied to 60 plants (Table 2). A control variant of 60 plants was sprayed the same way as preparations, only distilled water was used instead of active compound.

Table 1. Trade names, formulas and properties of tested PGRs

\begin{tabular}{|c|c|c|}
\hline Trade name & Formula & Properties \\
\hline $\begin{array}{c}\text { 2,3,5-Triiodobenzoic acid } \\
\text { (TIBA) }\end{array}$ & $\mathrm{C}_{7} \mathrm{H}_{3} \mathrm{I}_{3} \mathrm{O}_{2}$ & $\begin{array}{l}\text { Noncompetitive inhibitor } \\
\text { of polar auxin transport }\end{array}$ \\
\hline 4-CPPU & $\mathrm{C}_{12} \mathrm{H}_{10} \mathrm{ClN}_{3} \mathrm{O}$ & $\begin{array}{l}\text { Cytokinin plant } \\
\text { growth regulator }\end{array}$ \\
\hline $\begin{array}{l}\text { Naphthylphthalamic acid } \\
\text { (NPA) }\end{array}$ & $\mathrm{C}_{18} \mathrm{H}_{13} \mathrm{NO}_{3}$ & $\begin{array}{l}\text { Non competitive transport } \\
\text { inhibitor of auxin }\end{array}$ \\
\hline $\begin{array}{c}\text { Paclobutrazol } \\
\text { (PBZ) }\end{array}$ & $\mathrm{C}_{15} \mathrm{H}_{20} \mathrm{ClN}_{30}$ & $\begin{array}{l}\text { Plant growing retardant. } \\
\text { Antagonist of gibberellin }\end{array}$ \\
\hline $\begin{array}{l}\text { Epibrassinolide } \\
\text { (EBR) }\end{array}$ & $\mathrm{C}_{28} \mathrm{H}_{48} \mathrm{O}_{6}$ & $\begin{array}{l}\text { Promotion of cell expansion and cell elongation; } \\
\text { works with auxin to do so. } \\
\text { It has an unclear role in cell division and } \\
\text { cell wall regeneration }\end{array}$ \\
\hline $\begin{array}{l}\text { Ethephon } \\
\text { (Ethe.) }\end{array}$ & $\mathrm{C}_{2} \mathrm{H}_{6} \mathrm{ClO}_{3} \mathrm{P}$ & Plant growing regulator \\
\hline $\begin{array}{l}\text { Putrescine HCL } \\
\text { (Put) }\end{array}$ & $\mathrm{C}_{4} \mathrm{H}_{12} \mathrm{~N}_{2}{ }^{*} 2 \mathrm{HCl}$ & $\begin{array}{l}\text { Polyamine growth regulator affecting } \\
\text { the synthesis of macro-molecules, the activity of } \\
\text { macro-molecules, membrane permeability } \\
\text { and partial processes of mitosis and meiosis }\end{array}$ \\
\hline $\begin{array}{c}\text { 6-Benzylaminopurine } \\
\text { (6-BAP) }\end{array}$ & $\mathrm{C}_{12} \mathrm{H}_{11} \mathrm{~N}_{5}$ & Cytokinins \\
\hline $\begin{array}{c}\text { Gibberelic acid } 4+7 \\
\left(\mathrm{GA}_{4+7}\right)\end{array}$ & $\mathrm{C}_{19} \mathrm{H}_{24} \mathrm{O}_{5}, \mathrm{C}_{19} \mathrm{H}_{22} \mathrm{O}_{5}$ & Gibberellic acid \\
\hline
\end{tabular}


Table 2. Description of preparations, concentrations of active compounds and number of treated trees

\begin{tabular}{ccc}
\hline Preparation & Concentration & Number of treated trees \\
\hline Globaryl+EBR & $1 \%+0.001 \mathrm{ppm}$ & 60 \\
Globaryl & $1 \%$ & 60 \\
Progerbalin & $1.5 \%$ & 60 \\
GIBB & $0.5 \%$ & 60 \\
GIBB+4-CPPU & $0.5 \%+0.01 \%$ & 60 \\
Control & $\mathrm{x}$ & 60 \\
\hline TIBA+4-CPPU & $0.1 \%+0.01$ & 60 \\
NPA+4-CPPU & $0.001 \%+0.01 \%$ & 60 \\
TIBA+PBZ & $0.1 \%+0.1 \%$ & 60 \\
NPA+PBZ & $0.001 \%+0.1 \%$ & 60 \\
Etephon+Put+GA4+7 & $0.125 \%+0.2 \% 0.15 \%$ & 60 \\
Etephon+Put+6-BAP & $0.125 \%+0.2 \% 0.15 \%$ & 60 \\
\hline Progerbalin+4-CPPU+Put & $1.5 \%+0.01 \%+0.15 \%$ & 60 \\
\hline
\end{tabular}

Data collection and evaluation

The evaluation was carried out at the end of the vegetation period (in October) before lifting the nursery trees. Measurements were carried out by calliper and meter. To evaluate the efficacy of PGR preparations, following parameters were measured: tree height (the distance from the ground to the tip of the terminal shoot), trunk diameter above the budding site, trunk diameter under the first branching site, number of lateral shoots (in further text number of shoots) and the length of the lateral shoots (in further text - shoot length). Obtained data were subjected to statistical analyses in program STATISTICA 12 using a single and multi-factor analysis of variance (ANOVA). Subsequently, Duncan's test was used to calculate differences $(\mathrm{p}=0.05)$ between the treatments. To define the effective branching, a limit of $80 \%$ of branched trees for plum and $70 \%$ for sweet cherry trees was determined.

\section{Results}

Data of the 'Lipnická variety trees treated with TIBA+4-CPPU and NPA+4-CPPU were distorted as the trees grew in a field depression where more water retained. These variants of this variety were further not evaluated.

\section{Tree height}

The average height of 'Toptaste' trees ranged from 171.4

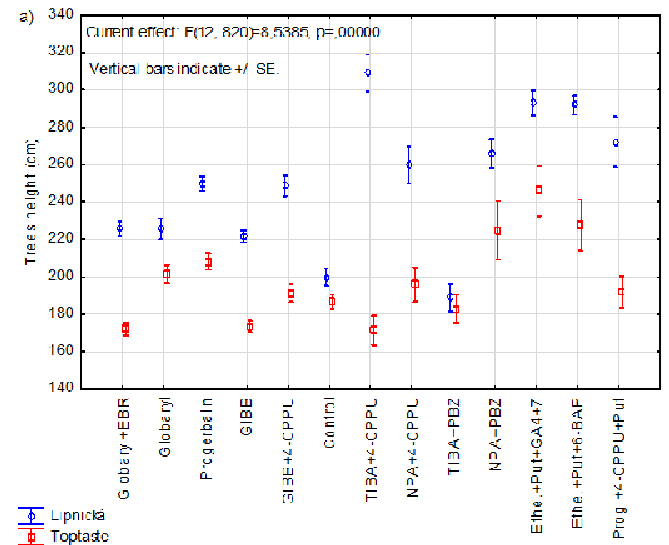

$\mathrm{cm}$ (TIBA+4-CPPU) to $246 \mathrm{~cm}$ (Ethe.+Put+GA4+7). Significantly higher trees compared to the control were measured after treatment with NPA+PBZ, Ethe.+Put+6BAP, and Ethe.+Put+GA $4+7$. At 'Lipnická' variety, the average height of the trees ranged from $189 \mathrm{~cm}$ (TIBA+PBZ) to $309 \mathrm{~cm}$ (TIBA+4-CPPU). Compared to the control, significantly higher trees were obtained in all variants except of TIBA+PBZ and GIBB plus variants (Table 3 and Fig. 1).

The average height of 'Kordia' trees ranged from 141.54 $\mathrm{cm}$ (TIBA+PBZ) to $181.5 \mathrm{~cm}$ (control) (Table 4). Compared to the control, all variants had lower trees, from which significantly lower trees, than of the control, were obtained after treatment with TIBA+PBZ, NPA+4CPPU, TIBA+4-CPPU, GIBB plus and GIBB +4-CPPU. At 'Celeste' variety, average tree height ranged from $78.3 \mathrm{~cm}$ (Ethe.+Put+6-BAP) to $158.36 \mathrm{~cm}$ (NPA+4-CPPU). Compared to the control, none of the variants had significantly higher trees, however, significantly lower trees were obtained in all variants except of Gibb+4-CPPU, $\mathrm{NPA}+\mathrm{PBZ}$, Progerbalin+4-CPPU+Put and NPA+4CPPU (Table 4 and Fig. 1).

On average, the highest trees were measured at 'Lipnická' variety $(235.2 \mathrm{~cm})$ and the lowest at variety 'Celeste' (129.72 cm). Significant differences in tree heights were calculated between all varieties (Table 5).

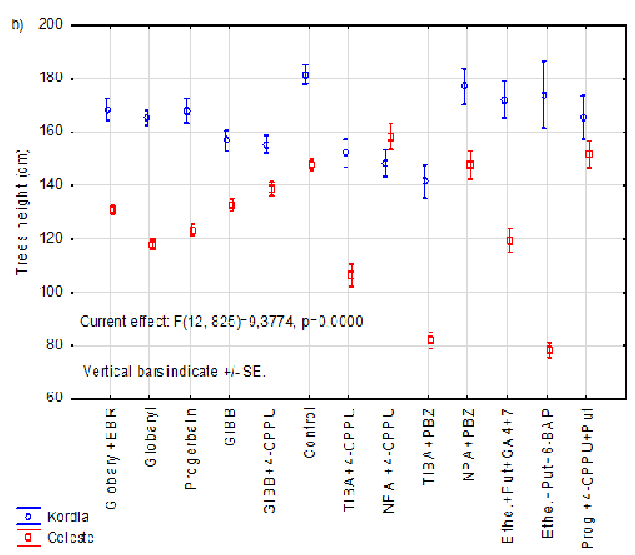

Fig. 1. The tree heights measured at all variants of a) plum varieties 'Toptaste' and 'Lipnická'; b) sweet cherry varieties 'Celeste' and 'Kordia' 
Table 3. Average values ( \pm standard errors) of parameters measured at plum varieties 'Toptaste' and 'Lipnická'. Grouping was calculated on the significance level of $p=0.5$

\begin{tabular}{|c|c|c|c|c|c|c|c|c|c|c|c|c|}
\hline \multirow[b]{2}{*}{ Preparation } & \multicolumn{6}{|c|}{ "Lipnická } & \multicolumn{6}{|c|}{ 'Toptaste' } \\
\hline & $\begin{array}{l}\text { Average tree } \\
\text { height }(\mathrm{cm})\end{array}$ & $\begin{array}{c}\text { Average } \\
\text { trunk } \\
\text { diameter } \\
\text { above the } \\
\text { budding } \\
(\mathrm{mm})\end{array}$ & $\begin{array}{l}\text { Average trunk } \\
\text { diameter } \\
\text { under } \\
\text { branching site } \\
\quad(\mathrm{mm})\end{array}$ & $\begin{array}{c}\text { Average } \\
\text { number of } \\
\text { shoots }\end{array}$ & $\begin{array}{c}\text { Average } \\
\text { length of the } \\
\text { shoots }(\mathrm{cm})\end{array}$ & $\begin{array}{c}\text { Branching } \\
\text { (\%) }\end{array}$ & $\begin{array}{l}\text { Average tree } \\
\text { height }(\mathrm{cm})\end{array}$ & $\begin{array}{c}\text { Average } \\
\text { trunk } \\
\text { diameter } \\
\text { above the } \\
\text { budding } \\
(\mathrm{mm})\end{array}$ & $\begin{array}{c}\text { Average } \\
\text { trunk } \\
\text { diameter } \\
\text { under } \\
\text { branching } \\
\text { site }(\mathrm{mm})\end{array}$ & $\begin{array}{c}\text { Average } \\
\text { number of } \\
\text { shoots }\end{array}$ & $\begin{array}{c}\text { Average } \\
\text { length of the } \\
\text { shoots }(\mathrm{cm})\end{array}$ & $\begin{array}{c}\text { Branching } \\
(\%)\end{array}$ \\
\hline Globaryl+EBR & $225.76 \pm 3.83 \mathrm{~cd}$ & $23.48 \pm 0.36 \mathrm{cde}$ & $15.09 \pm 0.31 \mathrm{bc}$ & $6.77 \pm 0.3 \mathrm{bcde}$ & $45.85+1.83 \mathrm{ab}$ & 96.61 & $172.03 \pm 3.21 \mathrm{a}$ & $19.96 \pm 0.36 \mathrm{a}$ & $16.36 \pm 0.37 \mathrm{ab}$ & $13.94 \pm 0.44 \mathrm{abc}$ & $45.73 \pm 1.23 \mathrm{ab}$ & 100 \\
\hline Globaryl & $225.59 \pm 5.5 \mathrm{~cd}$ & $21.89 \pm 0.48 \mathrm{abc}$ & $15.12 \pm 0.37 \mathrm{bc}$ & $7.10 \pm 0.5 \mathrm{cde}$ & $59.34 \pm 2.03 \mathrm{cde}$ & 84.75 & $201.49 \pm 4.88 \mathrm{bc}$ & $23.19 \pm 1.03 a b c$ & $18.16 \pm 0.41 \mathrm{bcd}$ & $15.05 \pm 1.03 a b c$ & $55.03 \pm 1.51 \mathrm{~cd}$ & 100 \\
\hline Progerbalin & $249.71 \pm 3.83 \mathrm{de}$ & $23.70 \pm 0.32 \mathrm{cde}$ & $16.96 \pm 0.3 \mathrm{cde}$ & $6.47 \pm 0.36 \mathrm{bcde}$ & $69.67 \pm 2.03 \mathrm{e}$ & 93.22 & $208.13 \pm 4.44 \mathrm{bcd}$ & $22.74 \pm 0.37 \mathrm{abc}$ & $18.50 \pm 0.34 \mathrm{~cd}$ & $12.96 \pm 0.43 \mathrm{abc}$ & $58.20 \pm 1.45 \mathrm{def}$ & 100 \\
\hline GIBB plus & $221.77 \pm 3.02 \mathrm{bc}$ & $22.50 \pm 0.29 \mathrm{bcd}$ & $14.23 \pm 0.25 b$ & $4.94 \pm 0.36 \mathrm{abcd}$ & $38.16 \pm 1.37 \mathrm{a}$ & 91.53 & $173.64 \pm 3.15 a$ & $19.87 \pm 0.31 \mathrm{a}$ & $15.18 \pm 0.23 \mathrm{a}$ & $13.79 \pm 0.39 \mathrm{abc}$ & $45.77 \pm 1.01 \mathrm{ab}$ & 100 \\
\hline $\mathrm{GIBB}+4-\mathrm{CPPU}$ & $248.64 \pm 5.52 \mathrm{de}$ & $24.28 \pm 0.28 \mathrm{def}$ & $16.13 \pm 0.31 \mathrm{bcde}$ & $5.25 \pm 0.29 \mathrm{abcd}$ & $60.82 \pm 2.97 \mathrm{cde}$ & 94.92 & $191.37 \pm 4.74 a b$ & $20.80 \pm 0.42 \mathrm{ab}$ & $16.30 \pm 0.37 \mathrm{ab}$ & $12.36 \pm 0.55 \mathrm{ab}$ & $51.85 \pm 1.41 \mathrm{bcd}$ & 100 \\
\hline Control & $199.96 \pm 4.38 \mathrm{ab}$ & $20.30 \pm 0.44 a$ & $12.52 \pm 0.35 \mathrm{a}$ & $7.74 \pm 0.52 \mathrm{e}$ & $34.80 \pm 2.14 a$ & 91.53 & $186.69 \pm 3.88 \mathrm{ab}$ & $22.72 \pm 0.44 a b c$ & $15.45 \pm 0.42 a$ & $12.00 \pm 0.6 \mathrm{ab}$ & $38.73 \pm 1.59 \mathrm{a}$ & 100 \\
\hline $\mathrm{TIBA}+4-\mathrm{CPPU}$ & $309.00 \pm 9.93 \mathrm{~g}$ & $26.50 \pm 1 \mathrm{~h}$ & $17.10 \pm 0.68 \mathrm{def}$ & $7.10 \pm 0.65 \mathrm{cde}$ & $69.70 \pm 5.5 \mathrm{e}$ & 100 & $171.40 \pm 7.7 \mathrm{a}$ & $20.45 \pm 0.79 \mathrm{ab}$ & $16.75 \pm 0.88 \mathrm{abc}$ & $13.60 \pm 1.11 \mathrm{abc}$ & $45.71 \pm 2.98 \mathrm{ab}$ & 100 \\
\hline $\mathrm{NPA}+4-\mathrm{CPPU}$ & $260.00 \pm 9.88 \mathrm{e}$ & $22.40 \pm 0.89 \mathrm{bcd}$ & $15.33 \pm 0.75 \mathrm{bcd}$ & $4.00 \pm 0.55 \mathrm{a}$ & $51.60 \pm 6.65 b \mathrm{~b}$ & 90 & $196.00 \pm 9.09 a b$ & $22.85 \pm 52 \mathrm{abc}$ & $19.60 \pm 0.5$ f́de & $14.70 \pm 0.49 \mathrm{abc}$ & $56.65 \pm 1.81 \mathrm{de}$ & 100 \\
\hline $\mathrm{TIBA}+\mathrm{PBZ}$ & $189.00 \pm 7.21 \mathrm{a}$ & $20.90 \pm 0.6 \mathrm{ab}$ & $14.40 \pm 0.32 \mathrm{~b}$ & $4.70 \pm 0.73 \mathrm{ab}$ & $52.19 \pm 4.4 \mathrm{bc}$ & 100 & $183.00 \pm 7.6 \mathrm{ab}$ & $23.70 \pm 1.19 b c$ & $18.90 \pm 0.83 \mathrm{de}$ & $14.00 \pm 0.76 \mathrm{abc}$ & $58.55 \pm 3.33 \mathrm{def}$ & 100 \\
\hline $\mathrm{NPA}+\mathrm{PBZ}$ & $266.00 \pm 7.77 \mathrm{e}$ & $23.75 \pm 0.82 \mathrm{cde}$ & $15.75 \pm 0.64 \mathrm{bcde}$ & $5.20 \pm 0.72 \mathrm{abcd}$ & $55.43 \pm 6.9 \mathrm{bcd}$ & 100 & $224.80 \pm 15.74 \mathrm{cde}$ & $24.95 \pm 0.9 c$ & $20.95 \pm 0.92 \mathrm{e}$ & $16.40 \pm 1.13 c$ & $64.42 \pm 4.34 \mathrm{ef}$ & 100 \\
\hline Etephon+Put+GA & $293.00 \pm 6.5 \mathrm{fg}$ & $25.50 \pm 0.47 \mathrm{fgh}$ & $18.80 \pm 0.5 \mathrm{~g}$ & $7.10 \pm 0.8 \mathrm{cde}$ & $67.07 \pm 3.29 \mathrm{de}$ & 100 & $246.00 \pm 13.37 \mathrm{e}$ & $24.40 \pm 1.02 \mathrm{c}$ & $19.80 \pm 0.94 \mathrm{de}$ & $15.50 \pm 1.16 \mathrm{bc}$ & $65.80 \pm 4.32 \mathrm{f}$ & 100 \\
\hline Etephon+Put+6-BAP & $292.00 \pm 5.12 \mathrm{fg}$ & $25.85 \pm 0.54 \mathrm{gh}$ & $17.55 \pm 0.54 \mathrm{fg}$ & $7.30 \pm 0.89 \mathrm{de}$ & $66.23 \pm 2.81 \mathrm{de}$ & 100 & $227.77 \pm 13.43 \mathrm{de}$ & $24.16 \pm 1.04 \mathrm{c}$ & $19.05 \pm 0.66 \mathrm{de}$ & $16.33 \pm 0.91 \mathrm{c}$ & $59.30 \pm 2.01 \mathrm{def}$ & 100 \\
\hline Progerbalin+4-CPPU+Put & $272.00 \pm 13.48 \mathrm{ef}$ & $22.40 \pm 0.77 \mathrm{bcd}$ & $15.25 \pm 1.21 \mathrm{bcd}$ & $4.87 \pm 0.78 \mathrm{abc}$ & $69.19 \pm 6.98 \mathrm{e}$ & 80 & $192.00 \pm 8.53 \mathrm{ab}$ & $20.65 \pm 1.1 \mathrm{ab}$ & $16.75 \pm 0.71 \mathrm{abc}$ & $11.70 \pm 0.91 \mathrm{a}$ & $48.57 \pm 4.56 \mathrm{bc}$ & 100 \\
\hline
\end{tabular}

Table 4. Average values ( \pm standard errors) of parameters measured at sweet cherry varieties 'Celeste' and 'Kordia'. Grouping was calculated on the significance level of $p=0.5$

\begin{tabular}{|c|c|c|c|c|c|c|c|c|c|c|c|c|}
\hline \multirow[b]{2}{*}{ Preparation } & \multicolumn{6}{|c|}{ 'Celeste' } & \multicolumn{6}{|c|}{ 'Kordia' } \\
\hline & $\begin{array}{l}\text { Average tree } \\
\text { height }(\mathrm{cm})\end{array}$ & $\begin{array}{c}\text { Average } \\
\text { trunk } \\
\text { diameter } \\
\text { above the } \\
\text { budding } \\
(\mathrm{mm})\end{array}$ & $\begin{array}{c}\text { Average } \\
\text { trunk } \\
\text { diameter } \\
\text { under } \\
\text { branching } \\
\text { site }(\mathrm{mm})\end{array}$ & $\begin{array}{c}\text { Average } \\
\text { number of } \\
\text { shoots }\end{array}$ & $\begin{array}{c}\text { Average } \\
\text { length of the } \\
\text { shoots }(\mathrm{cm})\end{array}$ & $\begin{array}{c}\text { Branching } \\
(\%)\end{array}$ & $\begin{array}{l}\text { Average tree } \\
\text { height }(\mathrm{cm})\end{array}$ & $\begin{array}{c}\text { Average trunk } \\
\text { diameter } \\
\text { above the } \\
\text { budding } \\
(\mathrm{mm})\end{array}$ & $\begin{array}{c}\text { Average } \\
\text { trunk } \\
\text { diameter } \\
\text { under } \\
\text { branching } \\
\text { site }(\mathrm{mm})\end{array}$ & $\begin{array}{c}\text { Average } \\
\text { number of } \\
\text { shoots }\end{array}$ & $\begin{array}{c}\text { Average } \\
\text { length of the } \\
\text { shoots }(\mathrm{cm})\end{array}$ & $\begin{array}{c}\text { Branching } \\
(\%)\end{array}$ \\
\hline Globaryl+EBR & $130.93 \pm 1.59$ def & $26.37 \pm 0.35 f$ & $13.65 \pm 0.36 \mathrm{abc}$ & $2.48 \pm 0.22 b$ & $30.31 \pm 1.78 \mathrm{ab}$ & 74.14 & $168.45 \pm 4.00 \mathrm{bcd}$ & $19.05 \pm 0.62 \mathrm{ab}$ & $17.26 \pm 0.65 \mathrm{ab}$ & $4.22 \pm 0.31 \mathrm{~d}$ & $59.42 \pm 1.93 \mathrm{abc}$ & 86.44 \\
\hline Globaryl & $117.89 \pm 1.92 \mathrm{bc}$ & $21.37 \pm 0.37 \mathrm{bc}$ & $13.63 \pm 0.39 \mathrm{abc}$ & $1.72 \pm 0.19 \mathrm{ab}$ & $36.90 \pm 2.07 \mathrm{abc}$ & 47.46 & $165.27 \pm 2.99 \mathrm{bcd}$ & $25.15 \pm 1.00 \mathrm{~d}$ & $17.48 \pm 0.41 \mathrm{ab}$ & $4.7 \pm 0.37 \mathrm{~d}$ & $53.17 \pm 1.36 \mathrm{ab}$ & 98.30 \\
\hline Progerbalin & $123.42 \pm 2.23 \mathrm{cde}$ & $22.47 \pm 0.34 \mathrm{~cd}$ & $13.07 \pm 0.61 \mathrm{ab}$ & $1.28 \pm 0.16 \mathrm{ab}$ & $38.45 \pm 3.63 \mathrm{abc}$ & 23.73 & $166.27 \pm 4.89 \mathrm{bcd}$ & $22.69 \pm 0.62 \mathrm{abcd}$ & $16.76 \pm 0.54 a b$ & $2.25 \pm 0.27 \mathrm{abc}$ & $66.26 \pm 4.15 c$ & 47.45 \\
\hline GIBB plus & $132.77 \pm 2.4 \mathrm{ef}$ & $23.78 \pm 0.41 \mathrm{de}$ & $15.14 \pm 0.76 \mathrm{bcd}$ & $1.00 \pm 0 \mathrm{a}$ & $36.57 \pm 3.79 \mathrm{abc}$ & 12.28 & $156.88 \pm 3.77 \mathrm{abc}$ & $23.06 \pm 1.05 \mathrm{bcd}$ & $15.51 \pm 0.45 \mathrm{ab}$ & $2.28 \pm 0.22 \mathrm{abc}$ & $55.21 \pm 1.99 \mathrm{abc}$ & 54.23 \\
\hline $\mathrm{GIBB}+4-\mathrm{CPPU}$ & $137.00 \pm 3.17 \mathrm{fg}$ & $26.74 \pm 0.47 \mathrm{f}$ & $16.14 \pm 0.53 \mathrm{~cd}$ & $1.95 \pm 0.20 \mathrm{ab}$ & $44.9 \pm 2.48 \mathrm{bc}$ & 36.84 & $155.33 \pm 3.24 \mathrm{abc}$ & $18.27 \pm 0.56 \mathrm{a}$ & $16.86 \pm 0.71 \mathrm{ab}$ & $4.00 \pm 0.57 \mathrm{bcd}$ & $59.26 \pm 2.36 \mathrm{abc}$ & 62.71 \\
\hline Control & $147.64 \pm 2.05 \mathrm{gh}$ & $26.57 \pm 0.41 \mathrm{f}$ & $16.08 \pm 0.53 \mathrm{~cd}$ & $1.32 \pm 0.11 \mathrm{ab}$ & $49.03 \pm 2.63 c$ & 43.86 & $181.50 \pm 3.57 \mathrm{~d}$ & $19.49 \pm 0.55 \mathrm{abc}$ & $17.57 \pm 0.60 \mathrm{ab}$ & $3.85 \pm 0.44 \mathrm{bcd}$ & $64.51 \pm 2.23 b c$ & 67.79 \\
\hline $\mathrm{TIBA}+4-\mathrm{CPPU}$ & $106.40 \pm 4.05 \mathrm{~b}$ & $22.90 \pm 1.05 \mathrm{~cd}$ & $\mathrm{x}$ & $\mathrm{x}$ & $\mathrm{x}$ & 0 & $153.70 \pm 4.99 \mathrm{abc}$ & $22.90 \pm 1.17 \mathrm{bcd}$ & $16.71 \pm 1.44 \mathrm{ab}$ & $1.66 \pm 0.33 a b$ & $53.40 \pm 2.42 \mathrm{abc}$ & 66.67 \\
\hline $\mathrm{NPA}+4-\mathrm{CPPU}$ & $158.36 \pm 4.65 \mathrm{~h}$ & $25.27 \pm 0.92 \mathrm{ef}$ & $15.20 \pm 1.15 \mathrm{bcd}$ & $2.00 \pm 0.44 \mathrm{ab}$ & $46.86 \pm 7.61 \mathrm{c}$ & 45.45 & $148.27 \pm 5.25 \mathrm{ab}$ & $21.27 \pm 1.49 \mathrm{abcd}$ & $15.88 \pm 1.26 \mathrm{ab}$ & $3.50 \pm 0.53 \mathrm{bcd}$ & $50.53 \pm 3.24 a$ & 81.82 \\
\hline $\mathrm{TIBA}+\mathrm{PBZ}$ & $82.20 \pm 3.07 \mathrm{a}$ & $20.10 \pm 0.01 \mathrm{ab}$ & $\mathrm{x}$ & $\mathrm{x}$ & $\mathrm{x}$ & 0 & $141.54 \pm 6.17 \mathrm{a}$ & $22.81 \pm 0.58 \mathrm{bcd}$ & $14.91 \pm 0.56 a$ & $2.45 \pm 0.36 \mathrm{abcd}$ & $50.89 \pm 4.23 a$ & 100 \\
\hline $\mathrm{NPA}+\mathrm{PBZ}$ & $147.72 \pm 5.34 \mathrm{gh}$ & $22.18 \pm 0.65 \mathrm{bcd}$ & $14.50 \pm 0.5 \mathrm{abcd}$ & $1.50 \pm 0.5 \mathrm{ab}$ & $30.25 \pm 4.75 a b$ & 25 & $162.80 \pm 17.95 \mathrm{abcd}$ & $25.70 \pm 0.80 \mathrm{~d}$ & $18.55 \pm 0.91 b$ & $4.12 \pm 0.81 \mathrm{~cd}$ & $57.46 \pm 2.00 \mathrm{abc}$ & 80 \\
\hline Etephon+Put+GA & $119.50 \pm 4.49 \mathrm{~cd}$ & $22.90 \pm 0.95 \mathrm{~cd}$ & $12.25 \pm 0.85 a$ & $1.50 \pm 0.28 \mathrm{ab}$ & $25.50 \pm 5.20 \mathrm{a}$ & 40 & $174.83 \pm 6.93 c d$ & $18.75 \pm 1.10 \mathrm{ab}$ & $17.00 \pm 1.87 \mathrm{ab}$ & $1.00 \pm 0 \mathrm{a}$ & $61.77 \pm 1.92 \mathrm{abc}$ & 45.45 \\
\hline Etephon+Put+6-BAP & $78.30 \pm 2.84 a$ & $18.20 \pm 0.84 \mathrm{a}$ & $\mathrm{x}$ & $\mathrm{x}$ & $\mathrm{x}$ & 0 & $174.00 \pm 12.54 \mathrm{~cd}$ & $25.09 \pm 0.69 \mathrm{~d}$ & $16.91 \pm 0.87 \mathrm{ab}$ & $3.33 \pm 0.68 \mathrm{bcd}$ & $56.39 \pm 3.95 a b c$ & 81.82 \\
\hline Progerbalin +4 -CPPU +Put & $151.05 \pm 5.08 \mathrm{~h}$ & $26.05 \pm 0.95 \mathrm{f}$ & $16.85 \pm 0.76 \mathrm{~d}$ & $2.00 \pm 0.30 \mathrm{ab}$ & $50.54 \pm 9.20 c$ & 70 & $165.18 \pm 7.33 \mathrm{bcd}$ & $23.54 \pm 0.98 \mathrm{~cd}$ & $17.00 \pm 1.36 \mathrm{ab}$ & $2.20 \pm 0.48 \mathrm{abc}$ & $62.16 \pm 7.54 a b c$ & 50 \\
\hline
\end{tabular}

Table 5. Overall average values ( \pm standard errors) of parameters measured at sweet cherry and plum varieties. Grouping was calculated on the significance level of $p=0.5$

\begin{tabular}{|c|c|c|c|c|c|}
\hline Variety & $\begin{array}{l}\text { Tree height } \\
(\mathrm{cm})\end{array}$ & $\begin{array}{l}\text { Trunk diameter above } \\
\text { the budding }(\mathrm{mm})\end{array}$ & $\begin{array}{l}\text { Trunk diameter under } \\
\text { branching site }(\mathrm{mm})\end{array}$ & $\begin{array}{c}\text { Number of } \\
\text { shoots }\end{array}$ & $\begin{array}{l}\text { Length of the shoots } \\
\qquad(\mathrm{cm})\end{array}$ \\
\hline \multicolumn{6}{|c|}{ Plum } \\
\hline 'Toptaste' & $191.61 \pm 1.74 c$ & $21.79 \pm 0.2 b$ & $17.01 \pm 0.15 b$ & $13.41 \pm 0.17 \mathrm{~d}$ & $50.48 \pm 0.62 b$ \\
\hline 'Lipnická' & $235.20 \pm 2.03 d$ & $22.88 \pm 0.15 c$ & $15.23 \pm 0.13 c$ & $6.27 \pm 0.15 c$ & $53.14 \pm 1.01 b$ \\
\hline \multicolumn{6}{|c|}{ Sweet Cherry } \\
\hline 'Kordia' & $164.90 \pm 1.44 b$ & $20.36 \pm 0.32 \mathrm{a}$ & $18.20 \pm 0.25 d$ & $3.56 \pm 0.14 b$ & $58.28 \pm 0.82 c$ \\
\hline 'Celeste' & $129.72 \pm 1.11 \mathrm{a}$ & $24.22 \pm 0.18 \mathrm{~d}$ & $14.53 \pm 0.21 \mathrm{a}$ & $1.84 \pm 0.09 \mathrm{a}$ & $38.60 \pm 1.17 \mathrm{a}$ \\
\hline
\end{tabular}

Note: Different letters between cultivars denote significant differences (Duncan test, $\mathrm{p}<0.05$ ).

\section{Trunk diameter above the budding site}

The trunk diameter above the budding site of 'Toptaste' variety ranged between $19.87 \mathrm{~mm}$ (GIBB) to $24.95 \mathrm{~mm}$ (NPA+PBZ). No significant differences were observed between the treatments and the control. At 'Lipnická' variety, the trunk diameter above the budding site ranged from $20.3 \mathrm{~mm}$ (control) to $26.5 \mathrm{~mm}$ (TIBA+4-CPPU). Compared to the control, significantly higher values were measured at all variants except for the TIBA+PBZ. and Globaryl. At both plum varieties the highest values of this parameter were measured in trees treated with
Ethe. + Put $+\mathrm{GA}_{4+7}$ and Ethe. + Put $+6-\mathrm{BAP}$ (Table 3).

The trunk diameter above the budding site of 'Kordia' variety ranged between $18.27 \mathrm{~mm}$ (Gibb+4-CPPU) to 25.7 $\mathrm{mm}(\mathrm{NPA}+\mathrm{PBZ})$. Compared to the control, significantly higher values were measured at variants treated with $\mathrm{NPA}+\mathrm{PBZ}$, Ethe.+Put+6-BAP and Globaryl. At 'Celeste' variety, the diameter of the trunk above the budding site ranged from $18.2 \mathrm{~mm}$ (Ethe.+Put.+6-BAP) to $26.74 \mathrm{~mm}$ $(\mathrm{Gibb}+4-\mathrm{CPPU})$, however no variants with significantly thicker trunks above the budding site were observed compared to the control (Table 4). 
966

On average, the highest values of trunk diameter above budding site were measured at 'Celeste' variety $(24.22 \mathrm{~mm})$ and the lowest at 'Kordia' variety $(20.36 \mathrm{~mm})$. Significant differences were calculated between all varieties (Table 5 ).

\section{Trunk diameter below the first branching site}

The trunk diameter below the first branching site of 'Toptaste' variety ranged from $15.18 \mathrm{~mm}$ (GIBB plus) to $20.95 \mathrm{~mm}$ (NPA+PBZ). Compared to the control, significantly higher values were measured at variants treated with Globaryl, Progerbalin, TIBA+PBZ, NPA+4-CPPU, $\mathrm{NPA}+\mathrm{PBZ}$, Ethe.+Put+GA $4+7$ and Ethe.+Put+6-BAP. At 'Lipnicka' variety, the trunk diameter below the first branching site ranged from $12.52 \mathrm{~mm}$ (control) to $18.8 \mathrm{~mm}$ (Ethe.+Put $\left.+\mathrm{GA}_{4+7}\right)$. All treated variants had significantly higher values of trunk diameters below the branching site as the control (Table 3).

The trunk diameter below the first branching site of 'Kordia' variety ranged from $14.91 \mathrm{~mm}$ (TIBA+PBZ) to $18.55 \mathrm{~mm}$ (NPA+PBZ). Significant differences were calculated only between variants treated with $\mathrm{NPA}+\mathrm{PBZ}$ and TIBA+PBZ. At 'Celeste' variety the trunk diameter below the first branching site ranged between $12.25 \mathrm{~mm}$ (Ethe.+Put+GA $4+7$ ) to $16.85 \mathrm{~mm}$ (Progerbalin+4CPPU+Put). None of the variants had significantly thicker trunks below the first branching site compared to the control (Table 4).

On average, the lowest values of trunk diameter below the first branching site were measured at 'Celeste' variety $(14.53 \mathrm{~mm})$ and the highest at 'Kordia' variety $(18.2 \mathrm{~mm})$. Significant differences were calculated between all varieties (Table 5).

\section{Branching}

All trees in all variants of 'Toptaste' variety were branched, whereas at 'Lipnická' variety at least $80 \%$ of the trees were branching in each variant. The lowest branching at 'Lipnická' variety was observed at treatment with Progerbalin+4-CPPU+Put (80\% of branched trees) see in Table 3.
At 'Kordia' variety Globaryl+EBR, Globaryl, NPA+4CPPU, TIBA+PBZ, NPA+PBZ and Ethe.+Put+6-BAP variants and at 'Celeste' variety only Globaryl+EBR and Progerbalin+4-CPPU+Put variants had more than $70 \%$ of trees branched. On the other hand, at 'Celeste' variety no branching was observed at trees treated with TIBA+4CPPU, TIBA+PBZ and Ethe.+Put+6-BAP (Table 4).

\section{Number and length of the shoots}

At 'Toptaste' variety, the average number of shoots ranged from 11.7 (Progerbalin+4-CPPU+Put) to 16.4 (NPA.+PBZ.) (Table 3). Significantly higher number of shoots compared to the control were recorded only at $\mathrm{NPA}+\mathrm{PBZ}$ and Ethe.+Put+6-BAP variants (Fig. 2). The average shoot length at 'Toptaste' variety ranged from 38.73 $\mathrm{cm}$ (control) to $65.8 \mathrm{~cm}$ (Ethe. + Put $\left.+\mathrm{GA}_{4+7}\right)$. Compared to the control, significantly longer shoots were measured at all variants except for TIBA+4-CPPU, Globaryl+ERB and GIBB plus (Fig. 3). The average number of shoots at 'Lipnická' variety ranged from 4.00 (NPA+4-CPPU) to 7.74 (control). None of the variants had higher number of shoots than the control, moreover, NPA+4-CPPU, TIBA+PBZ, Progerbalin+4-CPPU+Put, GIBB plus, $\mathrm{NPA}+\mathrm{PBZ}$ and GIBB+4-CPPU variants had significantly lower number of shoots than the control. The average shoot length of 'Lipnická' variety ranged from $34.80 \mathrm{~cm}$ (control) to $69.70 \mathrm{~cm}$ (TIBA + 4-CPPU). Compared to the control, significantly longer shoots were measured at all variants except for those treated with GIBB plus and Globaryl+EBR.

The average number of shoots at 'Kordia' variety ranged from 1.00 (Ethe.+Put+GA $4+7$ ) to 4.70 (Globaryl) (Table 4). When compared to the control, significant difference was calculated only at Etephon+Put $+\mathrm{GA}_{4+7}$, showing however significantly lower number of shoots than the control (Fig. $2)$. The average shoot length at 'Kordia' variety ranged between $50.53 \mathrm{~cm}$ (NPA+4-CPPU) and $66.26 \mathrm{~cm}$ (Progerbalin) (Table 4). No significantly higher differences were observed between the treatments and the control.
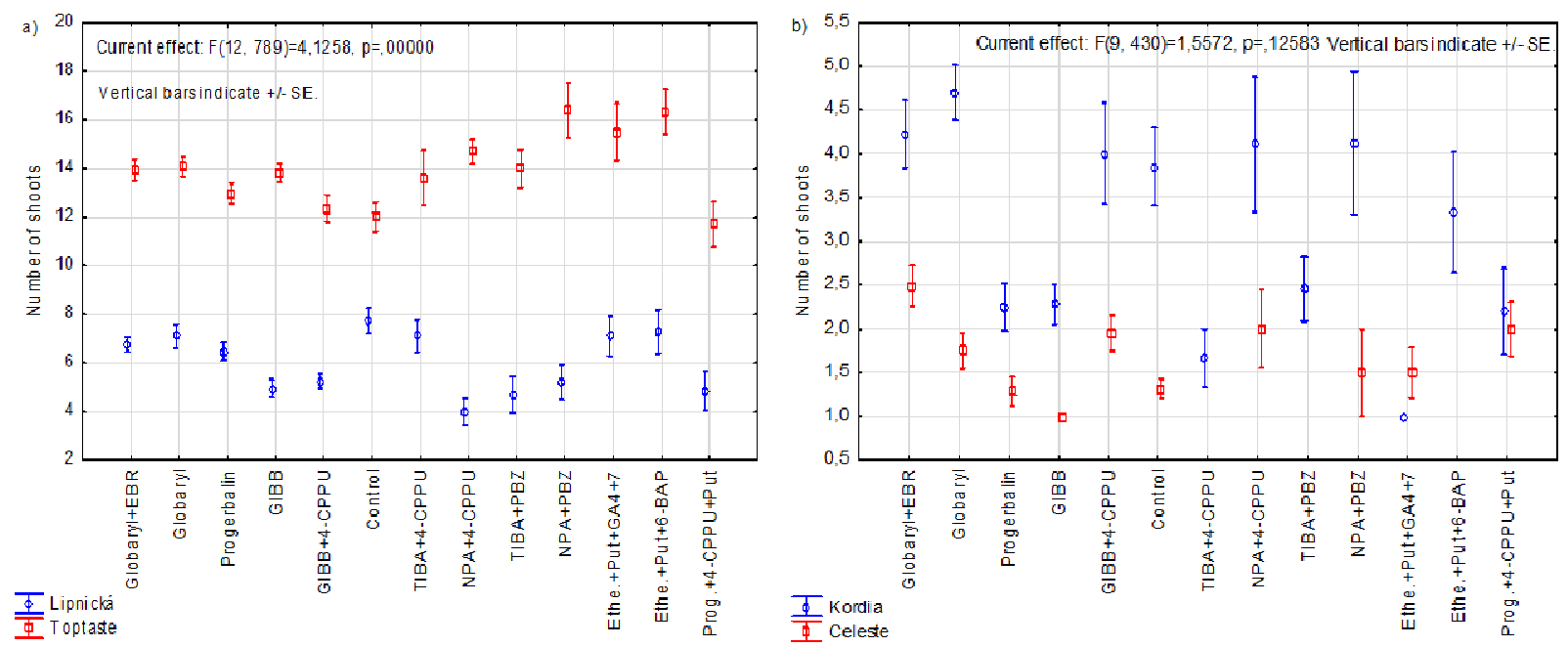

Fig. 2. The number of shoots counted at all variants of a) plum varieties 'Toptaste' and 'Lipnická'; b) sweet cherry varieties 'Celeste' and 'Kordia' 
On contrary, NPA+4-CPPU a TIBA+PBZ had significantly lower shoot length than the control (Fig. 3). At 'Celeste' variety no branching was observed after treatment with TIBA+4-CPPU, TIBA+PBZ, and Ethe.+put+6-BAP and therefore were not evaluated at number of shoots and shoot length parameters. At the rest variants, the average number of shoots ranged from 1.00 (GIBB plus) to 2.48 (Globaryl+EBR). Significant difference was found only between the variants treated with Globaryl+EBR and GIBB plus. The average shoot length at 'Celeste' variety ranged between $25.5 \mathrm{~cm}$ (Ethe.+Put+(GA ${ }_{4+7}$ ) and $50.54 \mathrm{~cm}$ (Progerbalin+4-CPPU+Put). None of the treated variants

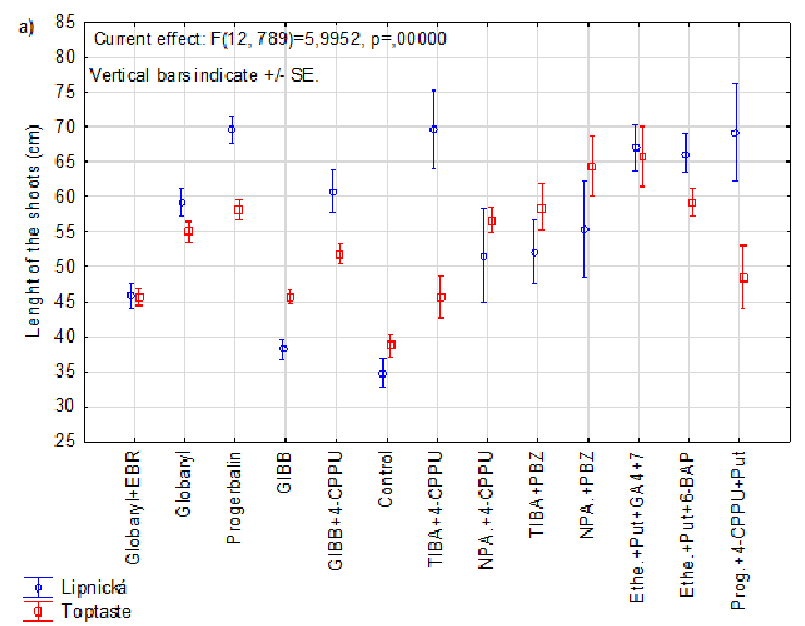

had significantly longer shoots than the control, however, significantly shorter shoots were measured at Globaryl+EBR, NPA+PBZ, and Ethe. + Put $+\mathrm{GA}_{4+7}$ variants.

On average, the highest number of shoots had the plum variety 'Toptaste' $(13,41)$ and the lowest sweet cherry variety 'Celeste' $(1,84)$. Significant differences were calculated between all varieties. On average, the longest shoots were measured at variety 'Kordia' $(58.28 \mathrm{~cm})$ and the shortest at 'Celeste' variety $(38.6 \mathrm{~cm})$. Significant differences were calculated between all varieties except for between 'Toptaste' and 'Lipnická' varieties (Table 5).

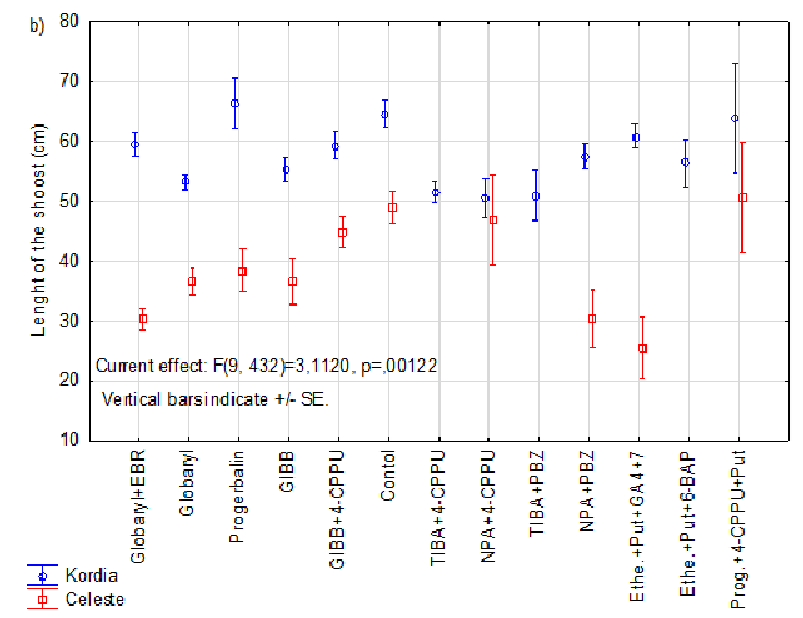

Fig. 3. The shoot lengths measured at all variants of a) plum varieties 'Toptaste' and 'Lipnická'; b) sweet cherry varieties 'Celeste' and 'Kordia'

\section{Discussion}

Managing the production of one-year-old trees is important for all fruit tree nurseries. Most of the plum varieties produce well-branched one-year-old trees even without application of PGRs. On the other hand, branching of some sweet cherry varieties is problematic and has been under research. Several publications (Hrotkó et al., 1999; Magyar and Hrotkó, 2005; Koyuncu and Yildrim, 2008) have studied branching promotion of sweet cherry trees, however, with low efficiency. Previous studies have shown positive effects of cytokinins (6-BAP) and gibberellins $\left(\mathrm{GA}_{4+7}\right)$ on branching of one-year-old trees (Elfving and Visser, 2007, 2009; Elfving et al., 2011). Except for these, several other PGRs were tested in this study to produce well-branched one-year-old nursery trees.

In the Czech Republic, according to the Decree $332 / 2006$ of Act 219/2003, the nursery tree must have at least 80 and $100 \mathrm{~cm}$ of height on a dwarfing and semivigorous to vigorous rootstocks, respectively. All tested plum trees in this study were grafted on a semi-vigorous St. Julien A rootstock and reached at least $150 \mathrm{~cm}$ of height. Sweet cherry trees were grown on a dwarfing rootstock Gisela 5 and except the variant Ethe.+Put+6-BAP (78.3 $\mathrm{cm}$ ) all trees reached the legal tree height of $80 \mathrm{~cm}$ (Table 4).

Tree height of sweet cherry varieties was negatively afected by all preparations, whereas at plum varieties this effect was observed only at 'Toptaste' treated with Globaryl+EBR and GIBB plus. An active compound of Globaryl and Progerbalin is 6-BAP, which decreases the tree hight by up to 10\% (Hrotkó et al., 2000; Dorić et al., 2016). The stronger effect of height decrease at sweet cherry varieties could be promoted by a significantly weaker growth (Table 5) and a higher number of applications of tested preparations compared to the plum varieties.

At both plum varieties, the highest nursery trees were obtained after treatment by Ethe.+Put+6-BAP, Ethe.+Put+GA4+7. Ethephon, an ethylene-releasing substance, is used to suppress the growth of terminal shoots due to an anti-gibberellin effect of ethylene (Lieberman, 1979). Putrescine, however, suppresses the effect of ethylene (Khan and Singh, 2010; Khan et al., 2008) and promotes growth in conjunctions with other phytohormones, for example with indole-3-butyric acid (Özkaya and Celik, 1994). In our case, the ethephon was applied in 10 times lower concentration than the effective concentration in Byers (1993) and its effect was probably revoked by putrescine, which led to the promotion of apical dominance. Another possible explanation could be the vigorous growth of both plum varieties which suppressed the effect of ethephon and at the same time promoted the growth through the synergism of putrescine with 6-BAP and $\mathrm{GA}_{4+7}$. The same could be the reason at sweet cherry variety 'Kordia', which showed similar results with these 
968

preparations. At 'Celeste' variety, Ethe.+Put+6-BAP, and Ethe. + Put $+\mathrm{GA}_{4+7}$ preparations negatively affected the tree heights, where trees treated with Ethe.+Put.+6-BAP preparation did not meet the standards for the nursery tree height $(80 \mathrm{~cm}$ according to the Decree 332/2006 of Act 219/2003). All varieties had lower tree heights after treatment with preparation containing 6-BAP (Ethe.+Put+6-BAP) compared to that containing $\mathrm{GA}_{4+7}$ (Ethe. + Put $+\mathrm{GA}_{4+7}$ ).

Growth of 'Celeste' variety was negatively affected also by TIBA+4-CPPU and TIBA+PBZ. Application of TIBA on stem and leaf reduces or completely interrupts apical dominance (Snyder, 1949; Panigrahi and Audus, 1966). A combination of poorly growing variety and TIBA, an auxin inhibitor, could negatively affect the height of treated 'Celeste' trees. The negative effect of TIBA was observed also on 'Toptaste' variety few days after the first application of TIBA+4-CPPU and TIBA+PBZ, however, due to vigorous growth, the negative effect was suppressed and at the evaluation period, no effect of TIBA was observed. At 'Lipnická' variety no significant difference was calculated between the control and the TIBA+PBZ variant too, however, trees treated with TIBA+PBZ had the smallest trees among all variants. Growth suppression of vigorous varieties is beneficial, however, it should be kept in mind that paclobutrazol is deposited in the plants for a longer time and could lead to unexpected growth reduction in the future (Jacyna et al., 1989).

The trunk diameter above budding site and below first branching site generally does not affect the quality of the nursery tree. On average, 'Lipnická' variety had significantly thicker trunks above the budding site than the 'Toptaste' variety. On contrary, significantly thicker trunks under the first branching site had the 'Toptaste' variety, which was however, branching by $13.22 \mathrm{~cm}$ higher than 'Lipnická' variety $(63.56 \mathrm{~cm})$ (data not shown).

Among all varieties, the highest trunk diameter above budding site was measured at poorly growing sweet cherry variety 'Celeste' $(24.22 \mathrm{~mm})$. At the same time, 'Celeste' variety had the lowest trunk diameter below the first branching site $(14.53 \mathrm{~mm})$ among all varieties. This result was probably caused by the weak growth of this variety. At sweet cherry varieties, the trunk diameters above the budding site varied considerably between the treatments, however, very similar results were obtained at both varieties at trunk diameters below the first branching site.

The thickest trunks were expected at paclobutrazolcontaining preparations $(\mathrm{PBZ}+\mathrm{TIBA}$ and $\mathrm{NPA}+\mathrm{PBZ})$ as paclobutrazol significantly increases trunk diameter (Werner, 1993). However, increased trunk diameters were observed only in few cases in this study. Possible cause of this result could be the concentration of the paclobutrazol in our preparations, being $1000 \mathrm{ppm}$, which in the work of Werner (1993) showed weaker effect on trunk diameter than at the concentration of $750 \mathrm{ppm}$.

According to the Decree 332/2006 of Act 219/2003, all branched nursery trees must have at least three shoots with a minimum length of $30 \mathrm{~cm}$. The number and also the quality of the shoots are important parameters of nursery trees (Zahn, 1996). Both plum varieties met these requirements. 'Toptaste' variety gave very good results in number and length of the shoots after treatment with Ethe.+Put+6BAP, Ethe. + Put $+\mathrm{GA}_{4+7}$ and $\mathrm{NPA}+\mathrm{PBZ}$ and very similar results were obtained at 'Lipnická' variety too. Higher number of shoots were counted after treatment by preparation containing 6-BAP (Ethe.+Put+6-BAP), which agrees with positive effect of 6-BAP on branching described in several studies (Hrotkó et al., 1999; Magyar and Hrotkó, 2005; Koyuncu and Yildrim, 2008). The longest shoots were measured at trees treated with preparation containing $\mathrm{GA}_{4+7}$ (Ethe.+Putre+ $\mathrm{GA}_{4+7}$ ), which agrees with the results of several authors (Schmidt et al., 2009; Atay and Koyuncu, 2016) where $\mathrm{GA}_{4+7}$ prolonged the length of shoots in different apple varieties by $12-138 \%$. In this study, the shoot length of the 'Toptaste' variety increased by $70 \%$ and at 'Lipnická' variety by $92.7 \%$ after treatment containing $\mathrm{GA}_{4+7}$ (Ethe.+Pu+GA ${ }_{4+7}$ ) compared to control. Good results of Ethe.+Putre+6-BAP, Ethe.+Putre+GA $\mathrm{GA}_{4+7}$ preparations could be enhanced by ethephon, which significantly promotes branching (Lieberman, 1979).

At both plum varieties, all variants had longer shoots than the control. Increase in the shoot length is beneficial since plums often produce fruits at the ends of short shoots. After fruit drop, the end of the shoot shrivels and a truncated shoot is formed, which results in its degradation.

Best results of branching of sweet cherry variety 'Kordia' were observed after treatment with preparations containing 6-BAP (Globaryl - 98.3\%, Globaryl+EBR - $86.44 \%$ and Ethe.+Putre.+6-BAP - 81.82\%) and with preparations $\mathrm{NPA}+4-\mathrm{CPPU}$ (81.82\%), TIBA+PBZ (100\%) and $\mathrm{NPA}+\mathrm{PBZ}(80 \%)$. Positive branching effect of preparations containing 6-BAP was expected, however, branching of 'Kordia' variety could be also promoted by suppression of apical dominance with auxin inhibitors (TIBA and NPA) in association with paclobutrazol, which has shown branching promotion in the work of Werner (1993).

Efficient branching ( $\geq 70 \%$ of branched trees) of the poorly growing 'Celeste' variety was achieved only at treatment with Globaryl+EBR (74.14 \%) and Progerbalin+4-CPPU+Put (70\%). Although the efficiency of branching in these two variants was sufficient, low number of shoots and their position on the trunk resulted in their poor quality. Strong apical dominance and the dwarfing rootstock could be the reasons of unsuccessful branching of the 'Celeste' variety. For efficient branching, the optimal ratio between the concentration of auxins and cytokinins (reduction of auxin and increase of cytokinin concentrations) (Klee and Lanahan, 1995) is necessary in the shoot tissues. Thus, careful selection, higher number of applications or adjusting the concentrations of PGRs will be necessary in order to achieve effective branching at 'Celeste' variety.

Interesting effect was observed in variants treated with PBZ+NPA, where paclobutrazole caused abnormal thickening and internode shortening of the terminal shoot in the application site. Similar effect of paclobutrazole was described by Werner (1993) too. Another interesting effect was observed at 'Toptaste' variety treated with 6-BAP in variants Globaryl and Globaryl+EBR, where second-order sylleptic shoots reaching $10 \mathrm{~cm}$ of length were formed. However, the asset of this effect is questionable and could be revoked by lower concentrations of 6-BAP. 


\section{Conclusions}

In conclusion, no significant results were observed at preparations containing NPA and 4-CPPU. An exception at NPA was NPA+PBZ preparation, which showed promising results in plum varieties. Here, PBZ could have greater effect on the trees than NPA or a synergistic effect between NPA and PBZ occurred. However, the long-term effect of PBZ on plants is questionable. Overall at plums, the treatments with Ethephone+Put $+\mathrm{GA}_{4+7}$ and Ethephone+Put+6-BAP significantly increased almost all measured parameters. Both sweet cherry varieties were sufficiently branching after treatments with Globaryl+EBR, however the lateral shoots of 'Celeste' sweer cherry variety were of low quality. The same effect was observed after treatment with Progerbalin+4CPPU+Put as well. Sweet cherry variety 'Kordia' branched very well after treatment with Globaryl and TIBA+PBZ too, however TIBA+PBZ, the lateral shoots after treatment with the latter preparation were of low quality. 'Kordia' trees after Globaryl treatment were branched in $94 \%$ of cases with more than four lateral shoots.

\section{Acknowledgements}

This research was supported by the Ministry of Agriculture of the Czech Republic under Grant NAZV/KUS project No QJ1510081.

\section{Conflicts of interest}

The authors declare that there are no conflicts of interest related to this article.

\section{References}

Atay AN, Koyuncu F (2016). Manipulating regular bearing in 'Golden Delicious'/M9 apple trees using $\mathrm{GA}_{4+} 7$ and ethephon. International Journal of Fruit Science 16(1):10-22.

Byers ER (1993). Controlling growth of bearing apple trees with ethephon. HortScience 28(11):1103-1105.

Dorić M, Keserović Z, Magazin N, Milić B, Miodragović M (2016). Enhancing feathering of one- and two-year-old nursery trees of'Granny Smith' apple cultivar using benzyladenine and gibberellins. Acta Horticulturae 1139:503-508.

Elfving DC, Dwayne BV, Henry JL (2011). Gibberellins stimulate lateral branch development in young sweet cherry trees in the orchard. International Journal of Fruit Science 11(1):41-54.

ElfvingDC, Visser DB (2006). Cyclanilide induces lateral branching in sweet cherry trees. HortScience 41(1):149-153.

Elfving DC, Visser DB (2007). Improving the efficacy of cytokinin applications for stimulation of lateral branch develipment in young sweet cherry trees in the orchard. HortScience 42(2):251-256.

Elfving DC, Visser DB (2009). Stimulation of lateral branch development in young sweet cherry trees in the orchard without bark injury. International Journal of Fruit Science 9(2):166-175.

Hrotkó K, Magyar L, Öri B (1999). Improved feathering on one-year-old 'Germersdorfi FL 45' sweet cherry trees in the nursery. Die
Gartenbauwissenschaft 64(2):75-78.

Hrotkó K, Magyar L, Ronay Z (2000). Improved feathering on apple nursery trees by BA application. ActaHorticulturae 514:113-122.

Jacyna T, Wood DES, Trappitt SM (1989). Application of paclobutrazol and Promalin $\left(\mathrm{GA}_{4+7}+\mathrm{BAP}\right)$ in the training of 'Bing' sweet cherry trees. NewZealand Journal ofCrop Horticultural Science 17(1):41-47.

Khan AS, Singh Z, Abbasi NA, Swinny E (2008). Pre- or post-harvest applications of putrescine and low temperature storage affect fruit ripening and quality of 'Angelino' plum. Journal of Science of Food and Agriculture 88(10):1686-1695.

Khan AS, Sinngh Z (2010). Pre-harvest application of putrescine influences Japanese plum fruit ripening and quality. Food Science and Technology International 16(1):53-64.

Klee HJ, Lanahan MB (1995). Transgenic plants in hormone biology. In: Davies PJ (Eds). Plant hormone. Dordrecht, Springer, Neaderland pp 340-353.

Koyuncu F, Yildirim AN (2008). Induction of lateral branching of '0900 Ziraat' sweet cherry in the nursery with 6-benzyladenine $+\mathrm{GA}_{4+7}$. Acta Horticulturae 795:391-394.

Lieberman M(1979). Biosynthesis and action of ethylene. Annual Review of Plant Physiology 30:533-591.

Looney N, Jackson D (2011). Use of bioregulators in fruit production. In: Jackson DI, Looney NE, Morley-Bunker M (Eds). Temperate and Subtropical Fruit Production (3rd ed) Cambridge University Press, United Kingdom pp 92-100.

Magyar L, Hrotkó K (2002). Effect of 6-Benzyladenine (BA) and gibberellic acid $\left(\mathrm{GA}_{4+7}\right)$ application on feathering of plum cultivars in nursery. Acta Horticulturae 577:345-349.

Magyar L, Hrotkó K (2005). Effect of BA (6-bentyladenine) and GA $\mathrm{F}_{47}$ on feathering of sweet cherry cultivars in the nursery. Acta Horticulturae 667:417-422.

Özkaya MT, Celik M (1994). The Effect of rooting environment and combination of auxin polyamine on the rooting ability of Turkish olive cultivars Gemlik andDomat. Acta Horticulturae 356:31-34.

Panigrahi DA, Audus L (1966). Apical dominance in Vicia faba. Annals of Botany 30(11):457-473.

Pedersen MK, Burton JD, Coble HD (2006). Effect of cyclanilide, ethephon, auxin transport inhibitors, and temperature on whole plant defoliation. CropScience 46(4):1666-1672.

Preston AP (1968). Pruning and rootstock as factors in the production of primary branches on apple trees.Journal of Horticultural Science 43(1):17-22.

Sazo MM, Robinson TL (2011). The use of plant growth regulators for branching of nursery trees in NY State.New York Fruit Quarterly 19(2):5-9.

Schmidt TR, Elfving DC, Mcferson JR, Whiting MD (2009). Crop load overwhelms effects of gibberellic acid and ethephon on floral initiation in apple. HortScience 44(7):1900-1906.

Snyder WE (1949). Some responses of plants to 2, 3, 5-triiodobenzoic acid. Plant Physiology 24(2):195-206.

Werner $H$ (1993). Influence of paclobutrazol on growth and leaf nutrient content of mango (cv. Blanco). Acta Horticulturae 341:225-231.

Zahn FG (1996). Close planting in relation to low orchard height. Horticultural Science 28(1-2):58-66. 